\title{
On Introducing Approximate Solution Methods in Theory of Elasticity
}

\author{
AUTAR KAW, SON HO \\ Department of Mechanical Engineering, University of South Florida, 4202 E Fowler Avenue, ENB-118, \\ Tampa, Florida 33620-5350
}

Received 14 October 2004; accepted 18 November 2005

\begin{abstract}
This work presents how approximate solution methods were introduced in a graduate level course of Theory of Elasticity. The three methods introduced are the finite difference method, the finite element method, and the boundary element method. All methods are exemplified by the problem of a thick-walled cylinder subject to internal pressure with an axisymmetric response. Choosing a single problem to introduce the three methods demonstrates accuracy and efficacy of each method. (c) 2006 Wiley Periodicals, Inc. Comput Appl Eng Educ 14: 120-134, 2006; Published online in Wiley InterScience (www.interscience.wiley.com); DOI $10.1002 /$ cae. 20070
\end{abstract}

Keywords: elasticity; finite difference method; finite element method; boundary element method

\section{INTRODUCTION}

This paper is the third segment in an effort by the first author to modernize the traditional Theory of Elasticity course. In 1993, symbolic algebra computational tools such as Macsyma [1] (substituted by Maple [2] in 2000) were introduced in the course to be able to solve problems that were physically more realistic but mathematically complex. Problems included classical problems such as the triangular wedge with uniform load on its edge, multilayered

Correspondence to A. Kaw (kaw@eng.usf.edu). (C) 2006 Wiley Periodicals Inc. cylinders with shrink fitting, stresses in a connecting rod, etc.

In 2000, this effort was followed by integrating a single research problem $[3,4]$ of assembling fulcrums of bascule bridges into the classroom [5]. This research problem had broad-based use in exemplifying multiple concepts such as interference fits, axisymmetric response problems, transformation of strains and stresses, comparison of failure theories, critical crack length aspects, and effect of temperature dependent thermoelastic properties.

In the last 40 years, the use of approximation solution methods to solve complex problems in engineering and science has grown significantly. The widespread availability of powerful digital computers 
and commercial computational software based on these approximation methods with efficient solution algorithms has made it practical. In this paper, we are introducing the student to approximate methods of solving elasticity problems. The purpose of this paper is to provide elementary background on how approximation solution methods work, while using a single example to illustrate the differences between the approaches, and discuss the accuracy and efficacy of the methods.

The single example chosen is the classical problem of a uniformly pressurized thick-walled cylinder with an axisymmetric response (Fig. 1). This problem is chosen since it is simple enough to have an analytical solution, but complex enough such that its approximate solutions can be generalized for problems that are more complicated.

The approximate solution methods introduced in this paper are finite difference method, finite element method, and boundary element method. The governing boundary-value differential equation, which is the basic equation for exact solution, is used directly by finite difference method (numerical approximation solution), while the finite element method (Rayleigh-Ritz method) is based on the concept of total potential energy, and boundary element method is based on boundary integral equation.

\section{THICK-WALL CYLINDER PROBLEM}

\section{Problem Definition}

Consider a thick-walled cylinder (material properties: Young's modulus $E$, Poisson's ratio $\nu$ ) of inner radius, $a$, and outer radius, $b$, subjected to uniform internal pressure, $p_{\mathrm{i}}$, and external pressure, $p_{\mathrm{o}}$, (Fig. 1). Find

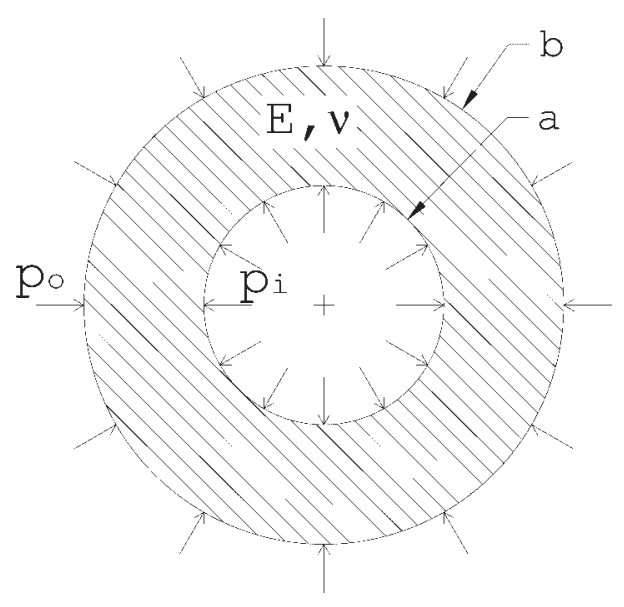

Figure 1 Pressured thick-wall cylinder problem. the radial displacement, $u$, and stress components (radial stress, $\sigma_{\mathrm{r}}$, and tangential stress, $\sigma_{\theta}$ ) in the cylinder. Plane stress state is assumed.

\section{Numerical Example Problem}

For demonstrating the use of approximate solution methods in solving the problem numerically, the following data is used: $a=0.25 \mathrm{~m}, b=0.5 \mathrm{~m}, p_{\mathrm{i}}=$ $200 \mathrm{MPa}, p_{\mathrm{o}}=0, E=207 \mathrm{GPa}, v=0.3$.

\section{Mathematical Formulation}

The solution of the thick-wall cylinder problem can be found by solving the equation of compatibility in polar coordinates, which is a fourth order partial differential equation of Airy stress function [6], or by using axisymmetry conditions to formulate the problem as a second order differential equation of displacement [7], or equivalent forms (potential energy, integral equation, etc.). The last approach is adopted in this paper, as it is direct and does not require inverse or semi-inverse solution methods $[6,7]$. The details of this approach are given in Reference [7] and the relevant formulas are summarized as follows. The radial strain, $\varepsilon_{\mathrm{r}}$, tangential strain, $\varepsilon_{\theta}$, in terms of radial displacement, $u$ are given as

$$
\begin{gathered}
\varepsilon_{\mathrm{r}}=\frac{\mathrm{d} u}{\mathrm{~d} r} \\
\varepsilon_{\theta}=\frac{u}{r}
\end{gathered}
$$

The radial stress, $\sigma_{\mathrm{r}}$, and tangential stress, $\sigma_{\theta}$, in terms of radial displacement, $u$, are given as

$$
\begin{gathered}
\sigma_{\mathrm{r}}=\frac{E}{1-\nu^{2}}\left(\frac{\mathrm{d} u}{\mathrm{~d} r}+\nu \frac{u}{r}\right) \\
\sigma_{\theta}=\frac{E}{1-\nu^{2}}\left(\nu \frac{\mathrm{d} u}{\mathrm{~d} r}+\frac{u}{r}\right)
\end{gathered}
$$

The governing equation for radial displacement, $u$, is given by

$$
\frac{\mathrm{d}^{2} u}{\mathrm{~d} r^{2}}+\frac{1}{r} \frac{\mathrm{d} u}{\mathrm{~d} r}-\frac{u}{r^{2}}=0
$$

Using Equations 3 and 4, the boundary conditions $\sigma_{\mathrm{r}}(a)=-p_{\mathrm{i}}$ and $\sigma_{\mathrm{r}}(b)=-p_{\mathrm{o}}$ can be rewritten as

$$
\begin{aligned}
& u^{\prime}(a)+\nu \frac{u(a)}{a}=-\frac{1-\nu^{2}}{E} p_{\mathrm{i}} \\
& u^{\prime}(b)+\nu \frac{u(b)}{b}=-\frac{1-\nu^{2}}{E} p_{\mathrm{o}}
\end{aligned}
$$


First, the exact solution is found, and then each approximate solution method is presented through solving the example problem. Nodal points chosen for the three approximate solution methods are uniformly spaced for convenience. Figure 2 shows how the nodal points and elements are numbered.

\section{Exact Solution}

The exact solution of displacement can be found directly by solving the governing differential equation, Equation 5, with associated boundary conditions, Equations 6 and 7 and then substituting it into Equations 3 and 4 give exact solution of stresses. The exact solutions [7] of radial displacement, radial stress, and tangential stress are obtained as

$$
\begin{gathered}
u=\frac{1-\nu}{E} \frac{\left(a^{2} p_{\mathrm{i}}-b^{2} p_{\mathrm{o}}\right) r}{b^{2}-a^{2}}+\frac{1+\nu}{E} \frac{\left(p_{\mathrm{i}}-p_{\mathrm{o}}\right) a^{2} b^{2}}{\left(b^{2}-a^{2}\right) r} \\
\sigma_{\mathrm{r}}=\frac{a^{2} p_{\mathrm{i}}-b^{2} p_{\mathrm{o}}}{b^{2}-a^{2}}-\frac{\left(p_{\mathrm{i}}-p_{\mathrm{o}}\right) a^{2} b^{2}}{\left(b^{2}-a^{2}\right) r^{2}} \\
\sigma_{\theta}=\frac{a^{2} p_{\mathrm{i}}-b^{2} p_{\mathrm{o}}}{b^{2}-a^{2}}+\frac{\left(p_{\mathrm{i}}-p_{\mathrm{o}}\right) a^{2} b^{2}}{\left(b^{2}-a^{2}\right) r^{2}}
\end{gathered}
$$

\section{Solution for Example Problem}

Substituting the numerical data into Equations 8-10, the exact solution for the example problem is

$$
\begin{gathered}
u=\left(0.2254 r+\frac{0.1047}{r}\right) \times 10^{-3} \\
\sigma_{\mathrm{r}}=\left(66.67-\frac{16.67}{r^{2}}\right) \times 10^{6} \\
\sigma_{\theta}=\left(66.67+\frac{16.67}{r^{2}}\right) \times 10^{6}
\end{gathered}
$$

Evaluating the solution at three nodal points (inner edge, $r=0.25 \mathrm{~m}$; mid-point, $r=0.375 \mathrm{~m}$; and outer edge, $r=0.5 \mathrm{~m}$ ) along the radial location for comparison, the resulted values are given in Table 1 .

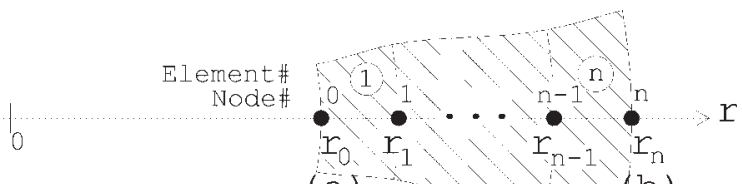
(a)

Figure 2 Numbering of nodal points and elements.
Table 1 Exact Solution Evaluated at Nodal Points

\begin{tabular}{lccc}
\hline$r(\mathrm{~m})$ & 0.25 & 0.375 & 0.5 \\
$u(\mathrm{~mm})$ & 0.4750 & 0.3637 & 0.3221 \\
$\sigma_{r}(\mathrm{MPa})$ & -200 & -51.85 & 0 \\
$\sigma_{\theta}(\mathrm{MPa})$ & 333.3 & 185.2 & 133.3 \\
\hline
\end{tabular}

\section{FINITE DIFFERENCE METHOD}

The finite difference method (FDM) is a numerical technique to solve differential equations [8]. It can be employed to solve this problem modeled as a boundary-value differential equation.

\section{Boundary-Value Differential Equation Formulation}

We recall here that the problem has been already formulated as a boundary-value differential equation, given by Equation 5 subject to the boundary conditions given by Equations 6 and 7 .

\section{Finite Difference Method}

The range of interest, $[a, b]$ is divided into $n$ segments by $n+1$ nodes numbered from 0 to $n$ (Fig. 2). For simple formulation, we use uniform nodal spacing, $h=(b-a) / n$. The derivatives of the unknown function (radial displacement) are presented as algebraic expressions of nodal coordinates. The relevant second-order approximation formulas are given in Table 2. More difference approximation formulas are available in [8].

Node 0: Approximate the first boundary condition, Equation 6, using forward difference formula

$$
\frac{1}{2 h}\left(-3 u_{0}+4 u_{1}-u_{2}\right)+\nu \frac{u_{0}}{a}=-\frac{1-\nu^{2}}{E} p_{\mathrm{i}}
$$

Node n: Approximate the second boundary condition, Equation 7, using backward difference formula

$$
\frac{1}{2 h}\left(u_{n-2}-4 u_{n-1}+3 u_{n}\right)+\nu \frac{u_{n}}{b}=-\frac{1-\nu^{2}}{E} p_{\mathrm{o}}
$$

Table 2 Finite Difference Formulas for First and Second Derivatives

\begin{tabular}{lccc}
\hline & $\begin{array}{c}\text { Forward } \\
\text { difference }\end{array}$ & $\begin{array}{c}\text { Backward } \\
\text { difference }\end{array}$ & $\begin{array}{c}\text { Central } \\
\text { difference }\end{array}$ \\
\hline$\left.\frac{\mathrm{d} f}{\mathrm{~d} x}\right|_{x=x_{0}}$ & $\frac{-3 f_{0}+4 f_{1}-f_{2}}{2 h} \frac{f_{-2}-4 f_{-1}+3 f_{0}}{2 h}$ & $\frac{-f_{-1}+f_{1}}{2 h}$ \\
$\left.\frac{\mathrm{d}^{2} f}{\mathrm{~d} x^{2}}\right|_{x=x_{0}}$ & & $\frac{f_{-1}-2 f_{0}+f_{1}}{h^{2}}$
\end{tabular}


Node $k(k=1 \ldots n-1)$ : Approximate the differential equation, Equation 5, using central difference formula

$$
\begin{aligned}
\frac{1}{h^{2}} & \left(u_{k-1}-2 u_{k}+u_{k+1}\right) \\
& +\frac{1}{r_{k}} \frac{1}{2 h}\left(-u_{k-1}+u_{k+1}\right)-\frac{u_{k}}{r_{k}^{2}}=0
\end{aligned}
$$

where

$$
r_{k}=a+k h
$$

From Equations 14-16, we get a system of $n+1$ equations of $n+1$ nodal displacements as

$$
\left\{\begin{array}{l}
\left(\frac{v}{a}-\frac{3}{2 h}\right) u_{0}+\left(\frac{2}{h}\right) u_{1}+\left(-\frac{1}{2 h}\right) u_{2}=-\frac{1-\nu^{2}}{E} p_{\mathrm{i}} \\
\vdots \\
\left(\frac{1}{h^{2}}-\frac{1}{2 h r_{k}}\right) u_{k-1}+\left(-\frac{2}{h^{2}}-\frac{1}{r_{k}^{2}}\right) u_{k}+\left(\frac{1}{h^{2}}+\frac{1}{2 h r_{k}}\right) u_{k+1}=0 \\
\vdots \\
\left(\frac{1}{2 h}\right) u_{n-1}+\left(-\frac{2}{h}\right) u_{n-1}+\left(\frac{\nu}{b}+\frac{3}{2 h}\right) u_{n}=-\frac{1-\nu^{2}}{E} p_{\mathrm{o}}
\end{array}\right.
$$

Solving this system of equations gives nodal displacement in the radial direction.

\section{Radial Stress and Tangential Stress}

From Equation 3 and Table 2, nodal radial stress at all nodal points can be presented in finite difference from as

$$
\left\{\begin{array}{l}
\sigma_{r, 0}=\frac{E}{1-\nu^{2}}\left(\frac{-3 u_{0}+4 u_{1}-u_{2}}{2 h}+\nu \frac{u_{0}}{a}\right) \\
\vdots \\
\sigma_{r, k}=\frac{E}{1-\nu^{2}}\left(\frac{-u_{k-1}+u_{k+1}}{2 h}+\nu \frac{u_{k}}{r_{k}}\right) \\
\vdots \\
\sigma_{r, n}=\frac{E}{1-\nu^{2}}\left(\frac{u_{n-2}-4 u_{n-1}+3 u_{n}}{2 h}+\nu \frac{u_{n}}{b}\right)
\end{array}\right.
$$

Similarly, from Equation 4 and Table 2, nodal tangential stress at all nodal points can be presented in finite difference form as

$$
\left\{\begin{array}{l}
\sigma_{\theta, 0}=\frac{E}{1-\nu^{2}}\left(\nu \frac{-3 u_{0}+4 u_{1}-u_{2}}{2 h}+\frac{u_{0}}{a}\right) \\
\vdots \\
\sigma_{\theta, k}=\frac{E}{1-\nu^{2}}\left(\nu \frac{-u_{k-1}+u_{k+1}}{2 h}+\frac{u_{k}}{r_{k}}\right) \\
\vdots \\
\sigma_{\theta, n}=\frac{E}{1-\nu^{2}}\left(\nu \frac{u_{n-2}-4 u_{n-1}-3 u_{n}}{2 h}+\frac{u_{n}}{b}\right)
\end{array}\right.
$$

Once the solution of nodal radial displacement has been found from Equation 17, nodal radial stress and nodal tangential stress are found by using Equations 18 and 19, respectively.

\section{Solution for Example Problem}

Consider the case $n=2$ with uniform spacing nodal points. The spacing between the nodes is $h=(b-a) /$ $n=(0.5-0.25) / 2=0.125$. The radial coordinates of the nodal points are $r_{0}=a=0.25, \quad r_{1}=0.375$, $r_{2}=b=0.5$.

Node 0: Approximate the first boundary condition, Equation 6, using forward difference formula

$$
\frac{1}{0.25}\left(-3 u_{0}+4 u_{1}-u_{2}\right)+0.3 \frac{u_{0}}{0.25}=-0.0008792
$$

Node 2: Approximate the second boundary condition, Equation 7, using backward difference formula

$$
\frac{1}{0.25}\left(u_{0}-4 u_{1}+3 u_{2}\right)+0.3 \frac{u_{2}}{0.5}=0
$$

Node 1: Approximate the differential equation, Equation 5, using central difference formula

$$
\begin{aligned}
& \frac{1}{0.125^{2}}\left(u_{0}-2 u_{1}+u_{2}\right)+\frac{1}{0.375} \frac{1}{0.25}\left(-u_{0}+u_{2}\right) \\
& -\frac{u_{1}}{0.375^{2}}=0
\end{aligned}
$$

From Equations 20-22, we obtained a system of algebraic equations of nodal radial displacement as

$$
\left\{\begin{aligned}
-10.80 u_{0}+16.00 u_{1}-4.000 u_{2} & =-0.0008792 \\
53.33 u_{0}-135.1 u_{1}+74.67 u_{2} & =0 \\
4.000 u_{0}-16.00 u_{1}+12.60 u_{2} & =0
\end{aligned}\right.
$$

Solving the above system of equations, the solution of nodal radial displacement is found as

$$
\left\{\begin{array}{l}
u_{0}=0.0005860 \\
u_{1}=0.0004309 \\
u_{2}=0.0003611
\end{array}\right.
$$

Employing Equations 18, 19, and 24, solutions of nodal radial stress and nodal tangential stress, respectively, are

$$
\begin{aligned}
& \left\{\begin{array}{l}
\sigma_{\mathrm{r}, 0}=-200 \times 10^{6} \\
\sigma_{\mathrm{r}, 1}=-126.2 \times 10^{6} \\
\sigma_{\mathrm{r}, 2}=0
\end{array}\right. \\
& \left\{\begin{array}{l}
\sigma_{\theta, 0}=425.2 \times 10^{6} \\
\sigma_{\theta, 1}=200 \times 10^{6} \\
\sigma_{\theta, 2}=149.5 \times 10^{6}
\end{array}\right.
\end{aligned}
$$


Table 3 Numerical Solution, Finite Difference Method $(n=2)$

\begin{tabular}{lccc}
\hline$r(\mathrm{~m})$ & 0.25 & 0.375 & 0.5 \\
$u(\mathrm{~mm})$ & 0.5860 & 0.4309 & 0.3611 \\
$\sigma_{r}(\mathrm{MPa})$ & -200 & -126.2 & 0 \\
$\sigma_{\theta}(\mathrm{MPa})$ & 425.2 & 200.0 & 149.5 \\
\hline
\end{tabular}

The numerical solution with $n=2$ of the example problem is summarized in Table 3.

Exact solution and numerical solutions with various values of number of nodal points, $n=2,3$, and 4, are given in Figure 3 for radial displacement and Figure 4 for radial stress.

The solution plots show that the approximate solutions approach the exact solution as the number of nodal points increases. The boundary conditions are also satisfied as they are incorporated directly in the formulation of the finite difference method.

\section{FINITE ELEMENT METHOD}

The Rayleigh-Ritz method can be viewed as a form of a finite element method (FEM) [9] where it reduces a continuous problem to a problem with finite number of degrees of freedom. The Rayleigh-Ritz method $[8,9]$ is based on the principle of stationary potential energy [9], which states: Among all admissible configurations of a conservative system, those that satisfy the equations of equilibrium make the potential energy stationary with respect to small variations of displacement. If the stationary condition is a minimum, the equilibrium state is stable.

Mathematically speaking, the Rayleigh-Ritz method is a variational method, based on the idea of finding a solution that minimizes a functional. For elasticity problems, the functional is the total potential energy. The solution must be admissible, that is, satisfying internal compatibility (e.g., continuity of displacement) and essential boundary conditions. For problems where displacements are primary unknowns, essential boundary conditions are prescriptions of displacement and non-essential boundary conditions are prescriptions of stress. Since the problem considered here (the thickwalled pressured cylinder problem, where primary unknown is radial displacement) has no prescription of displacement, there is no essential boundary condition.

\section{Potential Energy Formulation}

The cylinder is assumed in plane stress state to give strain energy density, $U_{0}$ as

$$
U_{0}=\frac{1}{2}\left(\sigma_{\mathrm{r}} \varepsilon_{\mathrm{r}}+\sigma_{\theta} \varepsilon_{\theta}\right)
$$

and using Equations 1-4, we get

$$
U_{0}=\frac{E}{2\left(1-\nu^{2}\right)}\left[\left(\frac{\mathrm{d} u}{\mathrm{~d} r}\right)^{2}+2 \nu\left(\frac{\mathrm{d} u}{\mathrm{~d} r}\right)\left(\frac{u}{r}\right)+\left(\frac{u}{r}\right)^{2}\right]
$$

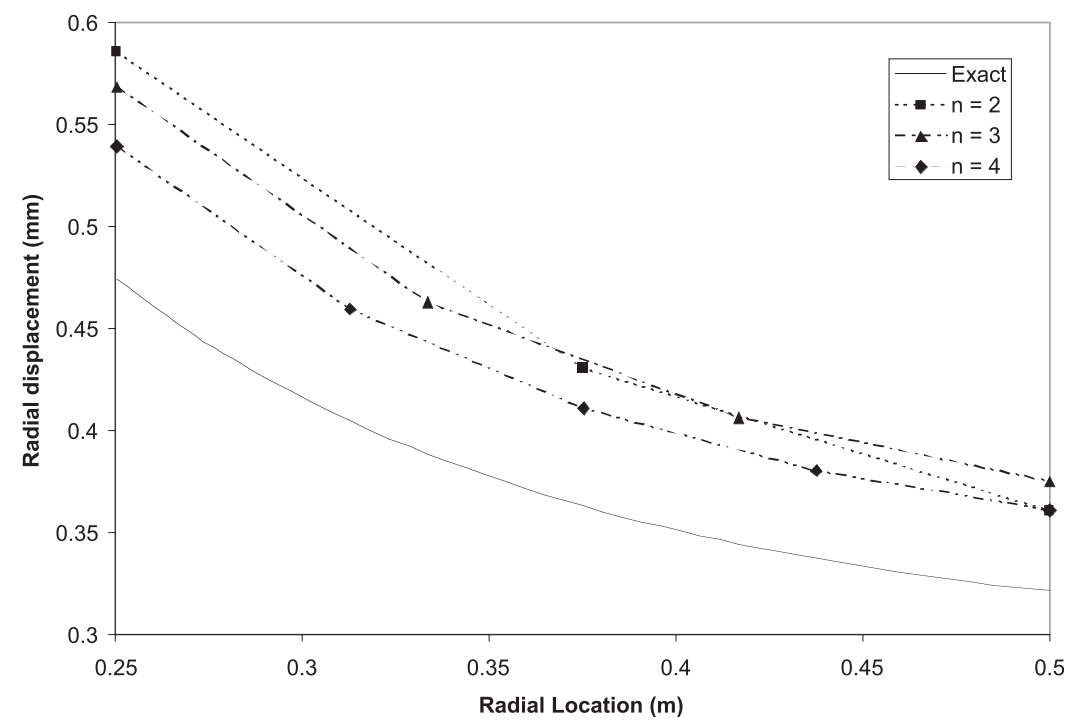

Figure 3 Radial displacement as a function of radial location (finite difference method). 


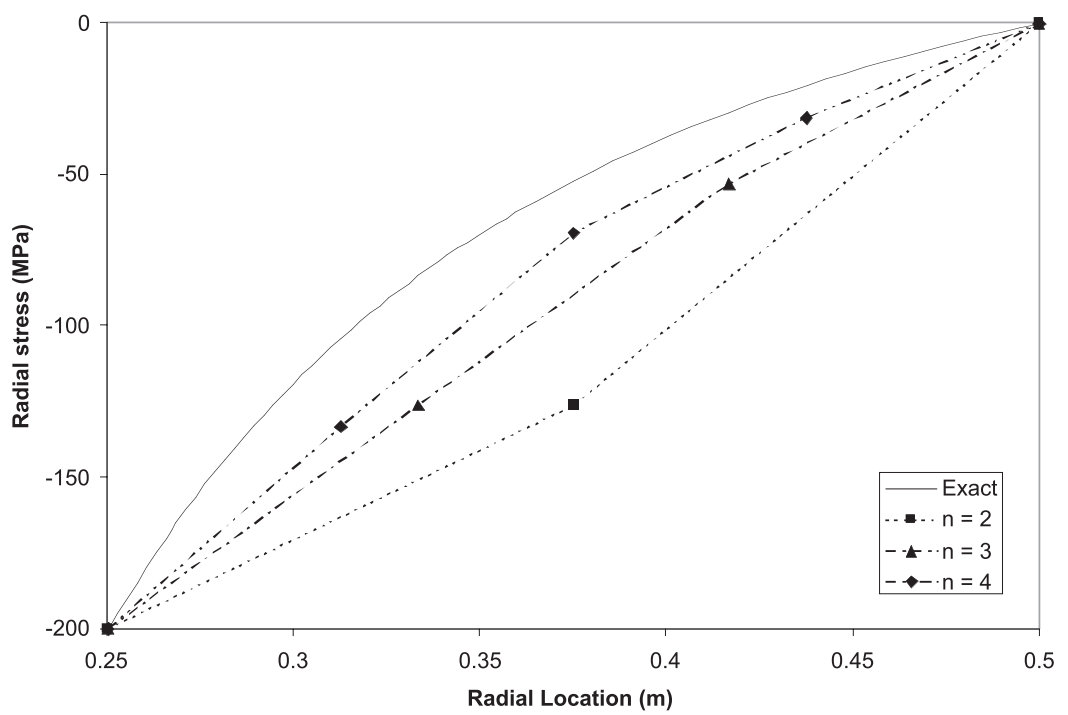

Figure 4 Radial stress as a function of radial location (finite difference method).

Total strain energy, $U$ of the cylinder is

$U=\int_{(V)} U_{0} \mathrm{~d} V=\int_{0}^{L} \int_{0}^{2 \pi} \int_{a}^{b} U_{0} r \mathrm{~d} r \mathrm{~d} \theta \mathrm{d} z=2 \pi L \int_{a}^{b} U_{0} r \mathrm{~d} r$

where

$L$, cylinder length

Work done, $W$ by external forces (internal and external pressures) is

$$
\begin{aligned}
W & =\int_{\left(S_{\mathrm{i}}\right)} p_{\mathrm{i}} u(a) \mathrm{d} s-\int_{\left(S_{\mathrm{o}}\right)} p_{\mathrm{o}} u(b) \mathrm{d} s \\
& =2 \pi a L p_{\mathrm{i}} u(a)-2 \pi b L p_{\mathrm{o}} u(b)
\end{aligned}
$$

where

$S_{i}$, inner cylinder surface

$S_{\text {o, }}$, outer cylinder surface

The total potential energy of the cylinder, $\Pi$ is found as

$\Pi=U-W=2 \pi L\left(\int_{a}^{b} U_{0} r \mathrm{~d} r-a p_{\mathrm{i}} u(a)+b p_{\mathrm{o}} u(b)\right)$

\section{Rayleigh-Ritz Method}

The Rayleigh-Ritz method can be outlined as follows

1. The potential energy of the system is given as $\Pi=\Pi\left(u^{\prime}, u, r\right)$.
2. Assume a trial solution of the form: $u=f$ $\left(r, C_{0}, C_{1}, \ldots, C_{m}\right)$ where $C_{i}$ 's $(i=0 . . m)$ are unknown parameters and $f$ is a known function. In this paper, we consider linear piecewise continuous functions.

3. Apply admissibility conditions to the trial solution. If there are $m-n$ admissibility conditions, we have $m-n$ equations of unknown parameters.

4. Solve the system of $m-n$ equations for $m-n$ unknowns $C_{n+1} \ldots C_{m}$, and then plug them back into the trial solution, we obtain a new trial solution that is admissible and has fewer unknowns ( $n$ unknowns) $u=f\left(r, C_{0}, C_{1}, \ldots, C_{n}\right)$.

5. Substitute the trial solution into the expression of potential energy.

6. The stationary condition for potential energy $\delta \Pi=0$ gives

$$
\left\{\frac{\partial \Pi}{\partial C_{i}}=0\right\}, \quad i=0 . . n
$$

7. Here we have a system of $n$ algebraic equations with $n$ unknowns. Solve this system of equations, we find the unknown parameters and thus the approximate solution for radial displacement.

8. Substitute the found solution for radial displacement into Equations 3 and 4 find the approximation solution for radial stress and tangential stress.

\section{Linear Piecewise Continuous Solution for Example Problem}

Consider the case of $n=2$ with uniform spacing nodal points. The step size for locating nodal points is 
calculated as $h=(b-a) / n=(0.5-0.25) / 2=0.125$. The radial coordinates of the nodal points are $r_{0}=a=0.25, r_{1}=0.375, r_{2}=b=0.5$.

The displacement field is assumed to be a piecewise continuous function of two linear segments as

$$
u= \begin{cases}C_{0}+C_{1} r, & 0.25 \leq r \leq 0.375 \\ C_{3}+C_{2} r, & 0.375 \leq r \leq 0.5\end{cases}
$$

To make the trial solution, Equation 33, admissible, it must be continuous at $r=0.375$, which means

$$
\begin{gathered}
C_{0}+0.375 C_{1}=C_{3}+0.375 C_{2}, \quad \text { or } \\
C_{3}=C_{0}+0.375 C_{1}-0.375 C_{2}
\end{gathered}
$$

The trial solution, Equation 33, then becomes

$u=\left\{\begin{array}{l}C_{0}+C_{1} r, \quad 0.25 \leq r \leq 0.375 \\ C_{0}+0.375 C_{1}-0.375 C_{2}+C_{2} r, \quad 0.375 \leq r \leq 0.5\end{array}\right.$

Substitute Equation 36 and the given numerical data into Equation 31, the total potential energy, $\Pi$ in the cylinder is found as

$$
\begin{aligned}
\Pi= & 2 \pi L\left(78.84 C_{0}^{2}+61.50 C_{0} C_{1}+12.42 C_{0} C_{2}\right. \\
& +16.15 C_{1}^{2}+4.659 C_{1} C_{2}+6.912 C_{2}^{2} \\
& \left.-0.05000 C_{0}-0.01250 C_{1}\right) \times 10^{9}
\end{aligned}
$$

The condition that the total potential energy $\Pi$ is stationary, $\left\{\frac{\partial \Pi}{\partial C_{0}}=0, \frac{\partial \Pi}{\partial C_{1}}=0, \frac{\partial \Pi}{\partial C_{2}}=0\right\}$ gives a system of algebraic equations of the unknown coefficients as

$$
\left\{\begin{array}{l}
157.7 C_{0}+61.50 C_{1}+12.42 C_{2}=0.05000 \\
61.50 C_{0}+32.31 C_{1}+4.659 C_{2}=0.01250 \\
12.42 C_{0}+4.659 C_{1}+13.82 C_{2}=0
\end{array}\right.
$$

The unknown coefficients are found as

$$
\left\{\begin{array}{l}
C_{0}=0.0006737 \\
C_{1}=-0.0008496 \\
C_{2}=-0.0003191
\end{array}\right.
$$

Substituting Equation 39 into Equation 36, the approximate solution for radial displacement is

$u= \begin{cases}0.0006737-0.0008496 r, & 0.25 \leq r \leq 0.375 \\ 0.0004748-0.0003191 r, & 0.375 \leq r \leq 0.5\end{cases}$

Substitute numerical data and displacement solution from Equation 40 into Equations 3 and 4, we find the radial and tangential stresses as

$$
\sigma_{\mathrm{r}}= \begin{cases}\frac{45.97}{r}-251.2, & 0.25<r<0.375 \\ \frac{32.40}{r}-94.38, & 0.375<r<0.5\end{cases}
$$

$$
\sigma_{\theta}= \begin{cases}\frac{153.2}{r}-251.2, & 0.25<r<0.375 \\ \frac{108.0}{r}-94.38, & 0.375<r<0.5\end{cases}
$$

The solution of the radial displacement is continuous, since we have forced the trial solution to be admissible from the beginning, while the solutions for stresses are discontinuous at the interior knot $(r=0.375)$ between the two segments (elements). To have reasonable results, in practice, the stress value at the interior knot is taken as the average of two stress values. The numerical solution with $n=2$ of the example problem is given in Table 4.

Exact solution and numerical solutions with various values of number of nodal points, $n=2,3$, and 4, are given in Figure 5 for radial displacement and Figure 6 for radial stress.

The solution plots show that the approximate solutions approach the exact solution as the number of piecewise continuous functions increases. However, they do not satisfy the boundary conditions of radial stress. The assumption of piecewise continuous solution as opposed to a continuous solution makes computation easier for high number of segments in the piecewise functions, but it has the drawback of the discontinuity of stresses at the interior knots of the piecewise continuous function.

\section{BOUNDARY ELEMENT METHOD}

The boundary element method (BEM) is a general numerical technique for solving boundary integral equations [10]. First, we need to formulate the problem as boundary integral equations.

\section{Boundary Integral Equation Formulation}

We recall here that the boundary value differential equation model of the problem as Equation 5 subject to the boundary conditions given by Equations 6 and 7 . Integrating both sides of Equation 5 with respect to $r$ from $a$ to $x$ gives

$$
\int_{a}^{x} \frac{\mathrm{d}^{2} u}{\mathrm{~d} r^{2}} d r+\int_{a}^{x} \frac{1}{r} \frac{\mathrm{d} u}{\mathrm{~d} r} \mathrm{~d} r-\int_{a}^{x} \frac{u}{r^{2}} \mathrm{~d} r=0
$$

Table 4 Numerical Solution, Finite Element Method $(n=2)$

\begin{tabular}{lccc}
\hline$r(\mathrm{~m})$ & 0.25 & 0.375 & 0.5 \\
$u(\mathrm{~mm})$ & 0.4613 & 0.3551 & 0.3152 \\
$\boldsymbol{\sigma}_{r}(\mathrm{MPa})$ & -67.35 & -68.32 & -29.58 \\
$\boldsymbol{\sigma}_{\theta}(\mathrm{MPa})$ & 361.7 & 175.5 & 121.6 \\
\hline
\end{tabular}




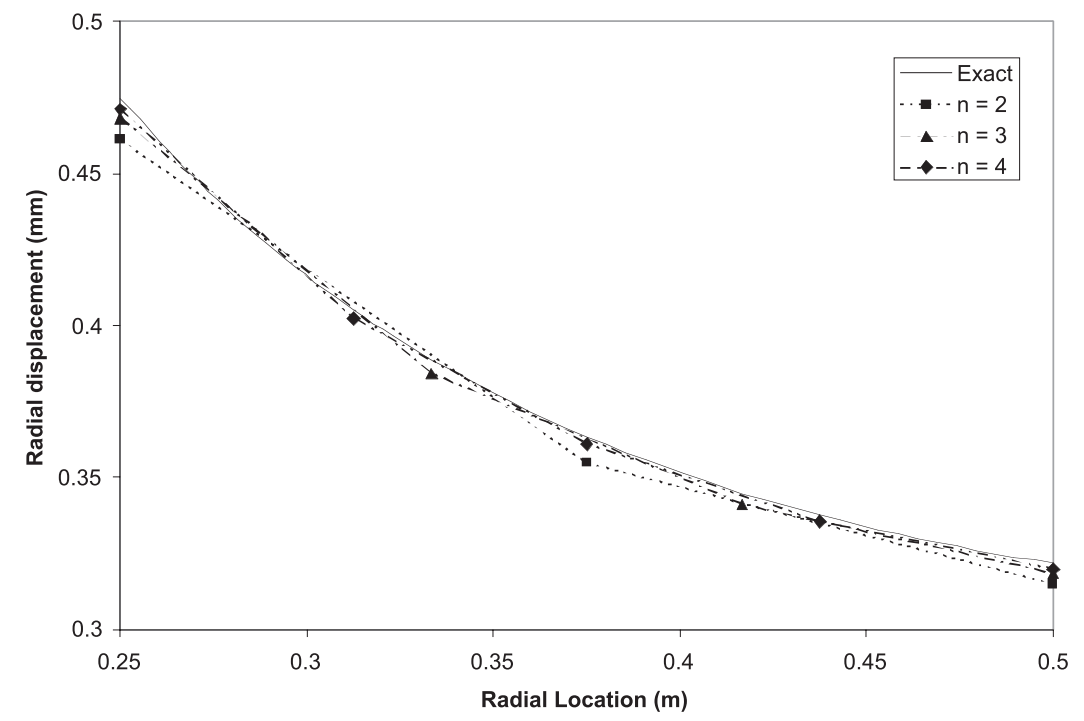

Figure 5 Radial displacement as a function of radial location (finite element method).

Manipulate and rewrite the first term on the left hand side of Equation 43 in reduced form as

$$
\begin{aligned}
& \int_{a}^{x} \frac{\mathrm{d}^{2} u}{\mathrm{~d} r^{2}} \mathrm{~d} r=\int_{a}^{x} \frac{\mathrm{d}}{\mathrm{d} r}\left(\frac{\mathrm{d} u}{\mathrm{~d} r}\right) \mathrm{d} r \\
& =\int_{a}^{x} \mathrm{~d}\left(u^{\prime}(r)\right)=\left[u^{\prime}(r)\right]_{a}^{x}=u^{\prime}(x)-u^{\prime}(a)
\end{aligned}
$$

Manipulate and rewrite the second term on the left hand of Equation 43 in reduced form as

$$
\begin{aligned}
\int_{a}^{x} \frac{1}{r} \frac{\mathrm{d} u}{\mathrm{~d} r} \mathrm{~d} r & =\int_{a}^{x} \frac{1}{r} \mathrm{~d} u=\left[\frac{u}{r}\right]_{a}^{x}-\int_{a}^{x} u\left(-\frac{1}{r^{2}}\right) \mathrm{d} r \\
& =\frac{u(x)}{x}-\frac{u(a)}{a}+\int_{a}^{x} \frac{u}{r^{2}} \mathrm{~d} r
\end{aligned}
$$

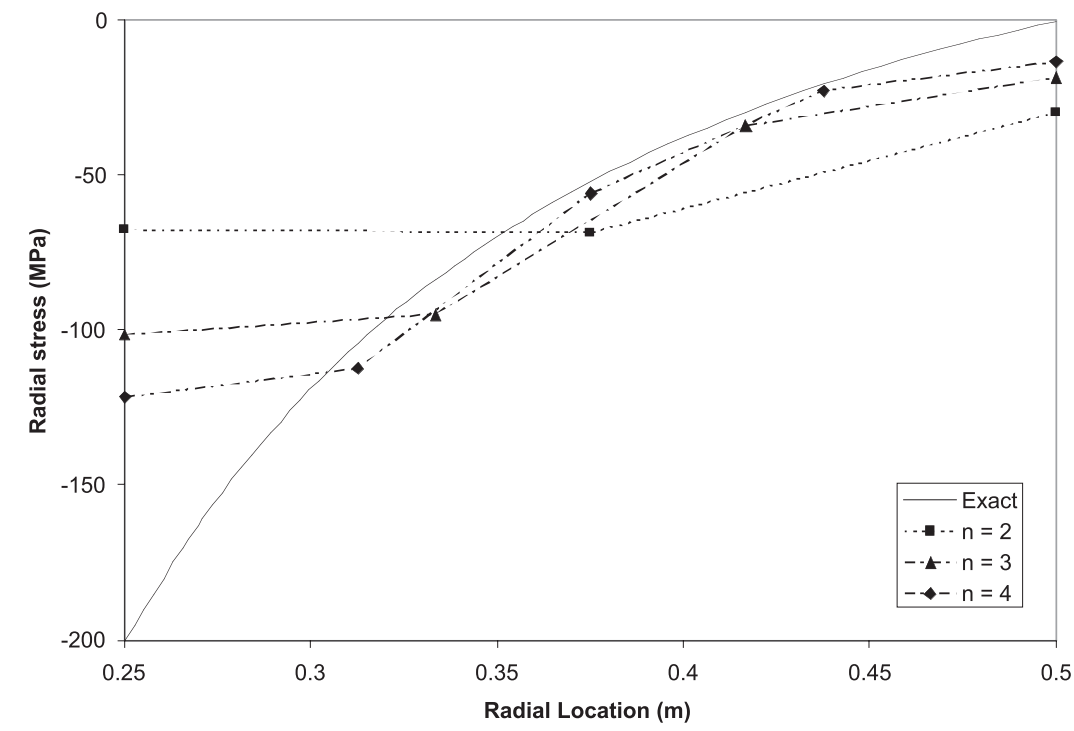

Figure 6 Solution of radial stress as a function of radial location (finite element method). 
Substituting Equations 44 and 45, Equation 43 becomes

$$
u^{\prime}(x)-u^{\prime}(a)+\frac{u(x)}{x}-\frac{u(a)}{a}=0
$$

Integrating both sides of Equation 46 with respect to $x$ from a to $r$ gives

$$
\begin{gathered}
\int_{a}^{r} u^{\prime}(x) \mathrm{d} x-\int_{a}^{r} u^{\prime}(a) \mathrm{d} x+\int_{a}^{r} \frac{u(x)}{x} \mathrm{~d} x-\int_{a}^{r} \frac{u(a)}{a} \mathrm{~d} x=0 \\
\Rightarrow u(r)-u(a)-u^{\prime}(a)(r-a) \\
\quad+\int_{a}^{r} \frac{u(x)}{x} \mathrm{~d} x-\frac{u(a)}{a}(r-a)=0, \quad \text { or } \\
u(r)-u^{\prime}(a)(r-a)-\frac{u(a)}{a} r+\int_{a}^{r} \frac{u(x)}{x} \mathrm{~d} x=0
\end{gathered}
$$

For Equation 47 be solved, we need to find $u(a)$ and $u^{\prime}(a)$. Evaluating Equation 46 at $x=b$ gives

$$
u^{\prime}(b)-u^{\prime}(a)+\frac{u(b)}{b}-\frac{u(a)}{a}=0
$$

Evaluating Equation 47 at $r=b$ gives

$$
u(b)-u^{\prime}(a)(b-a)-\frac{u(a)}{a} b+\int_{a}^{b} \frac{u(x)}{x} \mathrm{~d} x=0
$$

Equations 48 and 49, together with boundary conditions, Equations 6 and 7, form a system of four equations for four unknowns, namely $u(a), u^{\prime}(a), u(b)$, $u^{\prime}(b)$. These equations can be written in matrix form as

$$
\left[\begin{array}{cccc}
\frac{\nu}{a} & 0 & 1 & 0 \\
0 & \frac{\nu}{b} & 0 & 1 \\
-\frac{1}{a} & \frac{1}{b} & -1 & 1 \\
-\frac{b}{a} & 1 & a-b & 0
\end{array}\right]\left[\begin{array}{c}
u(a) \\
u(b) \\
u^{\prime}(a) \\
u^{\prime}(b)
\end{array}\right]=\left[\begin{array}{c}
-\frac{1-\nu^{2}}{E} p_{\mathrm{i}} \\
-\frac{1-\nu^{2}}{E} p_{\mathrm{o}} \\
0 \\
b \\
-\int_{a}^{b} \frac{u(x)}{x} \mathrm{~d} x
\end{array}\right]
$$

Solving Equation 50 simultaneously, we get

$$
\left\{\begin{array}{l}
u(a)=\frac{a}{\nu(b-a)}\left\{[(1-\nu) a+\nu b] P_{\mathrm{i}}-b P_{\mathrm{o}}-\int_{a}^{b} \frac{u(x)}{x} \mathrm{~d} x\right\} \\
u(b)=\frac{b}{\nu(b-a)}\left\{a P_{\mathrm{i}}-[(1-\nu) b+\nu a] P_{\mathrm{o}}-\int_{a}^{b} \frac{u(x)}{x} \mathrm{~d} x\right\} \\
u^{\prime}(a)=\frac{1}{b-a}\left\{b\left(P_{\mathrm{o}}-P_{\mathrm{i}}\right)+\int_{a}^{b} \frac{u(x)}{x} \mathrm{~d} x\right\} \\
u^{\prime}(b)=\frac{1}{b-a}\left\{a\left(P_{\mathrm{o}}-P_{\mathrm{i}}\right)+\int_{a}^{b} \frac{u(x)}{x} \mathrm{~d} x\right\}
\end{array}\right.
$$

where

$$
\left\{\begin{array}{l}
P_{\mathrm{i}}=\frac{1+\nu}{E} p_{\mathrm{i}}=\frac{p_{\mathrm{i}}}{2 G} \\
P_{\mathrm{o}}=\frac{1+\nu}{E} p_{\mathrm{o}}=\frac{p_{\mathrm{o}}}{2 G}
\end{array}\right.
$$

Substitute Equation 51 into Equation 47 and doing some algebraic manipulations as follows

$$
\begin{aligned}
& u(r)-\frac{r-a}{b-a}\left\{b\left(P_{\mathrm{o}}-P_{\mathrm{i}}\right)+\int_{a}^{b} \frac{u(x)}{x} \mathrm{~d} x\right\} \\
& -\frac{r}{\nu(b-a)}\left\{[(1-\nu) a+\nu b] P_{\mathrm{i}}-b P_{\mathrm{o}}\right. \\
& \left.-\int_{a}^{b} \frac{u(x)}{x} \mathrm{~d} x\right\}+\int_{a}^{r} \frac{u(x)}{x} \mathrm{~d} x=0 \\
& \Rightarrow \quad u(r)+\left(\frac{r}{\nu(b-a)}-\frac{r-a}{b-a}\right) \int_{a}^{b} \frac{u(x)}{x} \mathrm{~d} x \\
& +\int_{a}^{r} \frac{u(x)}{x} \mathrm{~d} x=\frac{r-a}{b-a} b\left(P_{\mathrm{o}}-P_{\mathrm{i}}\right) \\
& +\frac{r}{\nu(b-a)}\left\{[(1-\nu) a+\nu b] P_{\mathrm{i}}-b P_{\mathrm{o}}\right\}
\end{aligned}
$$

The resulted equation can be expanded and rearranged as

$$
\begin{aligned}
& u(r)+\frac{(1-\nu) r+\nu a}{\nu(b-a)} \int_{a}^{b} \frac{u(x)}{x} \mathrm{~d} x+\int_{a}^{r} \frac{u(x)}{x} \mathrm{~d} x \\
& =\frac{(1-\nu) r+\nu b}{\nu(b-a)} a P_{\mathrm{i}}-\frac{(1-\nu) r+\nu a}{\nu(b-a)} b P_{\mathrm{o}}
\end{aligned}
$$

or, in simpler form, as

$$
\begin{aligned}
& u(r)+\frac{\mu r+a}{b-a} \int_{a}^{b} \frac{u(x)}{x} \mathrm{~d} x+\int_{a}^{r} \frac{u(x)}{x} \mathrm{~d} x \\
& =\frac{\mu r+b}{b-a} a P_{\mathrm{i}}-\frac{\mu r+a}{b-a} b P_{\mathrm{o}}
\end{aligned}
$$

where

$$
\mu=\frac{1-\nu}{\nu}
$$

From Equation 53, splitting the first integral over interval $[a, b]$ into two integrals over intervals $[a, r]$ and $[r, b]$ gives

$$
\begin{aligned}
u(r) & +\frac{\mu r+a}{b-a}\left(\int_{a}^{r} \frac{u(x)}{x} \mathrm{~d} x+\int_{r}^{b} \frac{u(x)}{x} \mathrm{~d} x\right) \\
& +\int_{a}^{r} \frac{u(x)}{x} \mathrm{~d} x=\frac{\mu r+b}{b-a} a P_{\mathrm{i}}-\frac{\mu r+a}{b-a} b P_{\mathrm{o}}
\end{aligned}
$$


or, after simplifying,

$$
\begin{gathered}
u(r)+\frac{\mu r+b}{b-a} \int_{a}^{r} \frac{u(x)}{x} \mathrm{~d} x+\frac{\mu r+a}{b-a} \int_{r}^{b} \frac{u(x)}{x} \mathrm{~d} x \\
=\frac{\mu r+b}{b-a} a P_{\mathrm{i}}-\frac{\mu r+a}{b-a} b P_{\mathrm{o}}
\end{gathered}
$$

Now we combine the two integral terms into one such that

$$
\begin{gathered}
\int_{a}^{b} K(r, x) u(x) \mathrm{d} x=\frac{\mu r+b}{b-a} \int_{a}^{r} \frac{u(x)}{x} \mathrm{~d} x \\
\frac{\mu r+a}{b-a} \int_{r}^{b} \frac{u(x)}{x} \mathrm{~d} x, \quad a \leq r \leq b
\end{gathered}
$$

The function $K(r, x)$ is called the integral kernel in a Fredholm integral equation of second kind, which formulates the displacement problem to be shown later.

Consider the following cases:

i) $r=a$ : Equation 56 becomes

$$
\begin{aligned}
& \int_{a}^{b} K(a, x) u(x) \mathrm{d} x=\frac{\mu a+a}{b-a} \int_{a}^{b} \frac{u(x)}{x} \mathrm{~d} x \Rightarrow K(a, x) \\
& \quad=\frac{\mu a+a}{(b-a) x}, \forall x: a \leq x \leq b
\end{aligned}
$$

ii) $r=b$ : Equation 56 becomes

$$
\begin{aligned}
& \int_{a}^{b} K(r, x) u(x) \mathrm{d} x=\frac{\mu b+b}{b-a} \int_{a}^{b} \frac{u(x)}{x} \mathrm{~d} x \Rightarrow K(b, x) \\
& \quad=\frac{\mu b+b}{(b-a) x}, \forall x: a \leq x \leq b
\end{aligned}
$$

iii) $a<r<b$ : The function $K(r, x)$ that satisfies Equation 56 for all $x$, is defined as

$$
K(r, x)= \begin{cases}\frac{\mu r+b}{(b-a) x}, & a \leq x \leq r \\ \frac{\mu r+a}{(b-a) x}, & r<x \leq b\end{cases}
$$

Note that the special case $x=r$ can be combined to either the first condition $(a \leq x \leq r)$ or the second condition $(r \leq x \leq b)$. Here we use the former. $\Xi$ as

For simpler representations, we define a function

$\Xi(r, x)=\Xi(r, x, a, b)= \begin{cases}a, & (r=a) \text { and }(a \leq x \leq b) \\ b, & (r=b) \text { and }(a \leq x \leq b) \\ b, & (a<r<b) \text { and }(a \leq x \leq r) \\ a, & (a<r<b) \text { and }(r<x \leq b)\end{cases}$

\section{Displacement}

Equation 55 is the integral equation model of the displacement problem and can be presented as a Fredholm integral equation of second kind as

$$
u(r)+\int_{a}^{b} K(r, x) u(x) \mathrm{d} x=f(r), \quad a \leq r \leq b
$$

where

$$
\begin{gathered}
K(r, x)=\frac{\mu r+\Xi(r, x)}{(b-a) x} \\
f(r)=\frac{\mu r+b}{b-a} a P_{\mathrm{i}}-\frac{\mu r+a}{b-a} b P_{\mathrm{o}}
\end{gathered}
$$

in which, $P_{\mathrm{i}}, P_{\mathrm{o}}, \mu$, and $\Xi(r, x)$ are given by Equations 52, 54, and 57.

\section{Radial Stress}

The radial stress is given as

$$
\sigma_{r}=\frac{E}{1-\nu^{2}}\left(\frac{d u}{d r}+\nu \frac{u}{r}\right)=\hat{\Psi}_{R S}[u(r)]
$$

where $\hat{\Psi}_{R S}$ is the displacement to radial stress operator defined as

$$
\hat{\Psi}_{R S}=\frac{E}{1-\nu^{2}}\left(\frac{\partial}{\partial r}+\frac{\nu}{r}\right)
$$

Apply the operator $\hat{\Psi}_{R S}$ to both sides of the Equation 55 , we get

$$
\begin{aligned}
\sigma_{\mathrm{r}}(r)+ & \frac{E}{1-\nu^{2}}\left\{\frac{\mu(1+\nu) r+\nu b}{(b-a) r} \int_{a}^{r} \frac{u(x)}{x} \mathrm{~d} x\right. \\
+ & \left.\frac{\mu(1+\nu) r+\nu a}{(b-a) r} \int_{r}^{b} \frac{u(x)}{x} \mathrm{~d} x+\frac{u(r)}{r}\right\}=\frac{E}{1-\nu^{2}} \\
& \left\{\frac{\mu(1+\nu) r+\nu b}{(b-a) r} a P_{\mathrm{i}}-\frac{\mu(1+\nu) r+\nu a}{(b-a) r} b P_{\mathrm{o}}\right\}
\end{aligned}
$$

Rewrite the above equation, the integral equation for radial stress is obtained as

$$
\sigma_{\mathrm{r}}(r)=\frac{E}{1-\nu^{2}}\left\{f_{\mathrm{RS}}(r)-\frac{u(r)}{r}-\int_{a}^{b} K_{\mathrm{RS}}(r, x) u(x) \mathrm{d} x\right\}
$$

where

$$
K_{\mathrm{RS}}(r, x)=\frac{\mu(1+\nu) r+\nu \Xi(r, x)}{(b-a) r x}
$$




$$
f_{\mathrm{RS}}(r)=\frac{\mu(1+\nu) r+\nu b}{(b-a) r} a P_{\mathrm{i}}-\frac{\mu(1+\nu) r+\nu a}{(b-a) r} b P_{\mathrm{o}}
$$

\section{Tangential Stress}

The tangential stress is given as

$$
\sigma_{\theta}=\frac{E}{1-\nu^{2}}\left(\nu \frac{\mathrm{d} u}{\mathrm{~d} r}+\frac{u}{r}\right)=\hat{\Psi}_{\mathrm{TS}}[u(r)]
$$

where $\hat{\Psi}_{\mathrm{TS}}$ is the displacement to tangential stress operator defined as

$$
\hat{\Psi}_{\mathrm{TS}}=\frac{E}{1-\nu^{2}}\left(\nu \frac{\partial}{\partial r}+\frac{1}{r}\right)
$$

Apply the operator $\hat{\Psi}_{\mathrm{TS}}$ to both sides of the Equation 55 and manipulate the resulted equation similarly as for radial stress, the integral equation for tangential stress is found as

$$
\sigma_{\theta}(r)=\frac{E}{1-\nu^{2}}\left\{f_{\mathrm{TS}}(r)-\nu \frac{u(r)}{r}-\int_{a}^{b} K_{\mathrm{TS}}(r, x) u(x) \mathrm{d} x\right\}
$$

where

$$
\begin{gathered}
K_{\mathrm{TS}}(r)=\frac{\mu(1+\nu) r+\Xi(r, x)}{(b-a) r x} \\
f_{\mathrm{TS}}(r)=\frac{\mu(1+\nu) r+b}{(b-a) r} a P_{\mathrm{i}}-\frac{\mu(1+\nu) r+a}{(b-a) r} b P_{\mathrm{o}}
\end{gathered}
$$

\section{Multistep Method}

There are a few numerical methods for solving ordinary integral equations. The standard multistep method is the most straightforward one and is outlined as follows.
1. Consider a Fredholm integral equation of second kind as

$$
u(r)+\int_{a}^{b} K(r, x) u(x) \mathrm{d} x=f(r), \quad a \leq r \leq b
$$

2. The integral interval $(a, b)$ is divided into $n$ segments by $(n+1)$ points including two boundaries. These points are called collocation points and the process is called collocation. Here we use uniformly spaced collocation points, in which the $j$ th point has the radial coordinate as

$$
r_{j}=a+j h, \quad \text { where } h=\frac{b-a}{n}, j=0 . . n
$$

Note that $r_{0}=a, r_{n}=b$

3. The integral is approximated by utilizing any integral approximation rule, such as Simpson's rule, Trapezoidal rule, etc. associated with the above collocation points. For simplicity, we use Trapezoidal rule as

$$
\begin{aligned}
& \int_{a}^{b} K(r, x) u(x) \mathrm{d} x \\
& \approx \frac{h}{2}\left(K(r, a) u(a)+2 \sum_{j=1}^{n-1} K\left(r, x_{j}\right) u\left(x_{j}\right)+K(r, b) u(b)\right)
\end{aligned}
$$

4. The original integral equation, Equation 58, is now approximated by

$$
\begin{aligned}
u(r)+ & \frac{h}{2}\left(K(r, a) u(a)+2 \sum_{j=1}^{n-1} K\left(r, x_{j}\right) u\left(x_{j}\right)+K(r, b) u(b)\right) \\
& =f(r)
\end{aligned}
$$

5. Evaluate the approximate equation at $n+1$ collocation points (or at $r=r_{k}, k=0 . . n$ ), we find $n+1$ algebraic equations where the unknowns are the values of function $u(r)$ evaluated at $n+1$ collocation points:

$$
\left\{\begin{array}{l}
u(a)+\frac{h}{2} K(a, a) u(a)+\sum_{j=1}^{n-1} h K\left(a, r_{j}\right) u\left(r_{i}\right)+\frac{h}{2} K(a, b) u(b)=f(a) \\
\vdots \\
u\left(r_{k}\right)+\frac{h}{2} K\left(r_{k}, a\right) u(a)+\sum_{j=1}^{n-1} h K\left(r_{k}, r_{j}\right) u\left(r_{i}\right)+\frac{h}{2} K\left(r_{k}, b\right) u(b)=f\left(r_{k}\right) \\
\vdots \\
u(b)+\frac{h}{2} K(b, a) u(a)+\sum_{j=1}^{n-1} h K\left(b, r_{j}\right) u\left(r_{j}\right)+\frac{h}{2} K(b, b) u(b)=f(b)
\end{array}\right.
$$


Note that the index $k$ denotes the radius where the equation is evaluated at, while the index $j$ denotes the radius used in approximating the integral, but they both refer to the same set of collocation points.

6. Solve the system of equations to find the numerical solution for $u(r)$.

\section{Radial Stress and Tangential Stress}

Once the numerical solution for displacement has been found, the numerical solutions for radial stress and tangential stress can be found by using similar techniques of approximating integrals, noticing that $u\left(r_{j}\right)=u_{j}$ and $\sigma\left(r_{j}\right)=\sigma_{j}$, for the Equations 63 and 68, respectively, we get
The case of $n=2$ with uniform spaced nodal points is considered. The integral interval, [0.25, 0.5], is divided uniformly into two segments by three collocation points (including the two boundaries). The step size for locating nodal points is calculated as $h=(b-a) / n=(0.5-0.25) / 2=0.125$. The radial coordinates of the nodal points are $r_{0}=a=0.25$, $r_{1}=0.375, r_{2}=b=0.5$. The displacement values at collocation points are denoted as $u_{0}=u(0.25), u_{1}=$ $u(0.375), u_{2}=u(0.5)$.

Approximating Equation 78 using Trapezoidal rule, it becomes

$$
\begin{aligned}
u(r) & +[2.333 r+\Xi(r, 0.25)] u(0.25)+[3.111 r \\
& +1.333 \Xi(r, 0.375)] u(0.375)+[1.167 r \\
& +0.5 \Xi(r, 0.5)] u(0.5)=0.002931 r \\
& +0.0006280,0.25 \leq r \leq 0.5
\end{aligned}
$$

$$
\begin{gathered}
\sigma_{\mathrm{r}}(r)=\frac{E}{1-\nu^{2}}\left\{f_{\mathrm{RS}}(r)-\frac{u(r)}{r}-\frac{h}{2}\left(K_{\mathrm{RS}}(r, a) u_{0}+2 \sum_{j=1}^{n-1} K_{\mathrm{RS}}\left(r, r_{j}\right) u_{j}+K_{\mathrm{RS}}(r, b) u_{n}\right)\right\} \\
\sigma_{\theta}(r)=\frac{E}{1-\nu^{2}}\left\{f_{\mathrm{TS}}(r)-\nu \frac{u(r)}{r}-\frac{h}{2}\left(K_{\mathrm{TS}}(r, a) u_{0}+2 \sum_{j=1}^{n-1} K_{\mathrm{TS}}\left(r, r_{j}\right) u_{j}+K_{\mathrm{TS}}(r, b) u_{n}\right)\right\}
\end{gathered}
$$

Evaluate these two equations at $n+1$ collocation points, noticing that $u\left(r_{k}\right)=u_{k}$ and $\sigma\left(r_{k}\right)=\sigma_{k}$, we find the numerical solutions for radial stress and tangential stress.

\section{Solution for Example Problem}

The integral equation for the numerical example are found by substituting the given numerical data into Equations 58-60 and obtained as

$$
\begin{aligned}
u(r)+ & \int_{0.25}^{0.5} \frac{9.333 r+4 \Xi ;(r, x)}{x} u(x) d x \\
& =0.002931 r+0.0006280,0.25 \leq r \leq 0.5
\end{aligned}
$$

Evaluate Equation 79 at the three collocation points; we got a system of three equations

$$
\left\{\begin{array}{l}
1.833 u_{0}+1.111 u_{1}+0.4167 u_{2}=0.001361 \\
1.375 u_{0}+2.833 u_{1}+0.5625 u_{2}=0.001727 \\
1.667 u_{0}+2.222 u_{1}+1.833 u_{2}=0.002093
\end{array}\right.
$$

The solution of nodal displacement is found as

$$
\left\{\begin{array}{l}
u_{0}=0.0004783 \\
u_{1}=0.0003122 \\
u_{2}=0.0003286
\end{array}\right.
$$

Substitute the given numerical data into Equations 63-65, we find the integral equation for radial stress as

$$
\sigma_{r}(r)=\left(0.8667+\frac{0.04286}{r}-\frac{227.5 u(r)}{r}-30.33 \int_{0.25}^{0.5} \frac{91 r+9 \Xi(r, x)}{r x} u(x) d x\right) \times 10^{9}
$$


The integral in Equation 82 is approximated by using Trapezoidal rule as

$$
\begin{aligned}
& \int_{0.25}^{0.5} \frac{91 r+9 \Xi(r, x)}{r x} u(x) \mathrm{d} x \\
= & \frac{22.75 r+2.250 \Xi(r, 0.25)}{r} u(0.25) \\
& +\frac{30.33 r+3 \Xi(r, 0.375)}{r} u(0.375) \\
& +\frac{11.38 r+1.125 \Xi(r, 0.5)}{r} u(0.5)
\end{aligned}
$$

Substituting Equation 83 into Equation 82 and then evaluating the resulting equation at three collocation points, we find the numerical solution for radial stress.

Similarly, substituting the given numerical data into Equations 66-68, we found the integral equation for tangential stress as

$$
\sigma_{\theta}(r)=\left(0.8667+\frac{0.1429}{r}-\frac{68.24 u(r)}{r}-30.33 \int_{0.25}^{0.5} \frac{91 r+30 \Xi(r, x)}{r x} u(x) \mathrm{d} x\right) \times 10^{9}
$$

The integral in Equation 84 is approximated by using Trapezoidal rule as

$$
\begin{aligned}
& \int_{0.25}^{0.5} \frac{91 r+30 \Xi(r, x)}{r x} u(x) d x \\
= & \frac{22.75 r+7.500 \Xi(r, 0.25)}{r} u(0.25) \\
& +\frac{30.33 r+10.00 \Xi(r, 0.375)}{r} u(0.375) \\
& +\frac{11.38 r+3.750 \Xi(r, 0.5)}{r} u(0.5)
\end{aligned}
$$

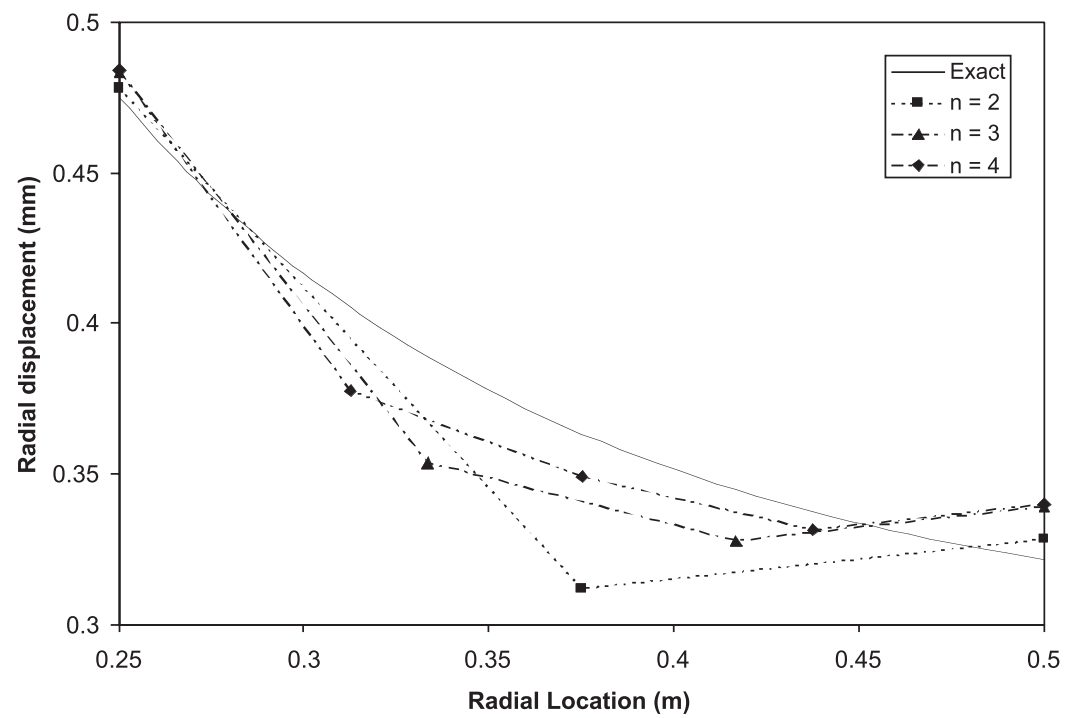

Figure 7 Radial displacement as a function of radial location (boundary element method).
Table 5 Numerical Solution, Boundary Element

\begin{tabular}{lccc}
\hline$r(\mathrm{~m})$ & 0.25 & 0.375 & 0.5 \\
$u(\mathrm{~mm})$ & 0.5860 & 0.4309 & 0.3611 \\
$\sigma_{r}(\mathrm{MPa})$ & -200 & -27.91 & 0 \\
$\sigma_{\theta}(\mathrm{MPa})$ & 336.0 & 164.0 & 136.0 \\
\hline
\end{tabular}

Substitute Equation 85 into Equation 84 and then evaluate the resulting equation at three collocation points, we find the numerical solution for hoop stress. The results are summarized in Table 5.

Exact solution and numerical solutions with various values of number of nodal points, $n=2,3$, and 4, are given in Figure 7 for radial displacement and Figure 8 for radial stress. Method $(n=2)$

\section{CONCLUSIONS}

Three approximation solution methods have been introduced through a single application example that can help teaching the basic ideas of these methods.

The solution plots show that the approximate solutions approach the exact solution as the number of collocation points increases. The boundary conditions are also satisfied.

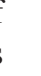




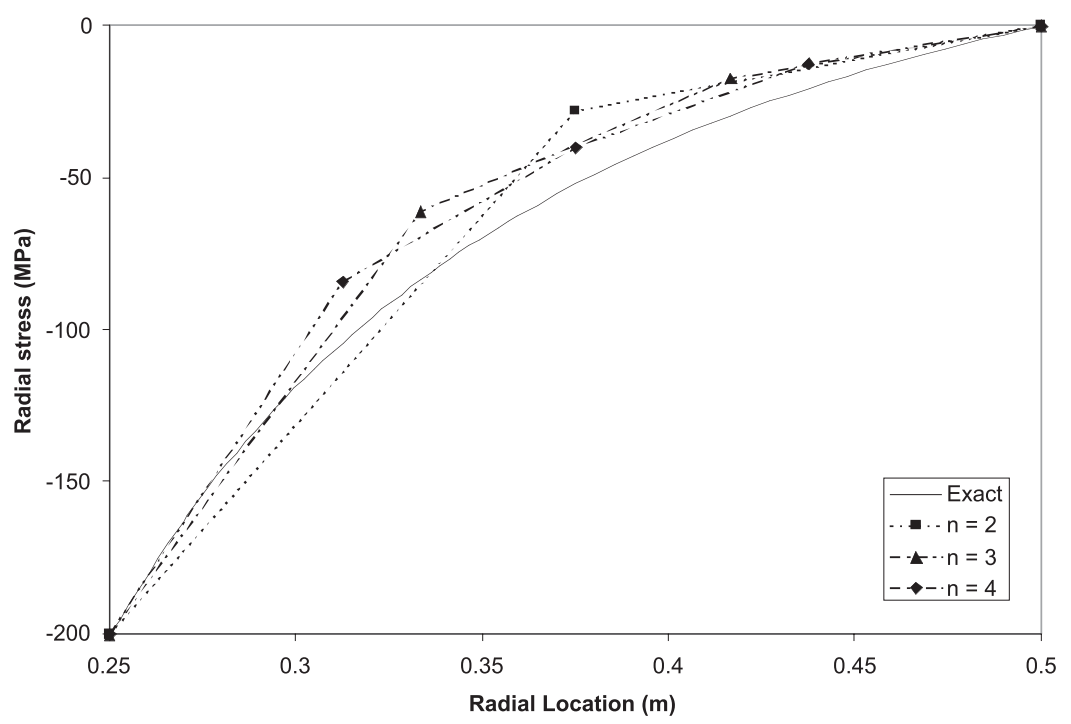

Figure 8 Radial stress as a function of radial location (boundary element method).

Based on the examples, the student can apply the solving schemes for this problem with higher degree approximations, or different trial solutions, or different rules of approximating derivatives and integrals. They can also attempt other physical problems with the same techniques. Some of these problems include a two-dimensional beam with a rectangular crosssection and concentrated axial load to illustrate St. Venant's principle. The Maple programs for the three methods are available at http://www.eng.usf.edu/ $\sim$ kaw/class/elasticity/

\section{ACKNOWLEDGMENTS}

The symbolic and numerical computations for this paper were performed by using Maple ${ }^{\mathrm{TM}}$. Maple and Maple 9 are registered trademarks of Waterloo Maple Inc. This material is based upon work supported partially by the National Science Foundation under Grant No. 0126793 and 0341468. Any opinions, findings, and conclusions or recommendations expressed in this material are those of the author(s) and do not necessarily reflect the views of the National Science Foundation.

\section{REFERENCES}

[1] Macsyma. See http://www.symbolicnet.org/systems/ macsyma.html.

[2] Maple 9.0, Advancing Mathematics. See http://www. maplesoft.com.

[3] G. H. Besterfield, S. Nichani, A. K. Kaw, and T. Eason, Full-scale testing of trunnion-hub-girder assemblies for bascule bridges, ASCE J Bridge Eng 8 (2003), 204-211.

[4] G. H. Besterfield, A. K. Kaw, S. Nichani, B. Ratnam, T. Cherukara, and M. Denninger, Assembly procedures of a trunnion-hub-girder for bascule bridges, Theor Appl Fracture Mech 40 (2003), 123-134.

[5] A. K. Kaw, G. H. Besterfield, and S. Nichani, Integrating a research problem in a course in applied elasticity, Int J Mech Eng Educ 32 (2004), 232-242.

[6] S. P. Timoshenko, and J. N. Goodier, Theory of elasticity, McGraw-Hill, New York, 1970.

[7] A. C. Ugural, and S. K. Fenster, Advanced strength and applied elasticity, 3rd ed., Prentice-Hall PTR, New York, 1995.

[8] A. P. Boresi, and K. P. Chong, Approximate solution methods in engineering mechanics, Elsevier Applied Science, New York, 1991.

[9] R. D. Cook, D. S. Malkus, and M. E. Plesha, Concepts and applications of finite element analysis, 3rd ed., Wiley, New York, 1989.

[10] W. S. Hall, The boundary element method, Kluwer Academic Publishers, New York, 1994. 


\section{BIOGRAPHIES}

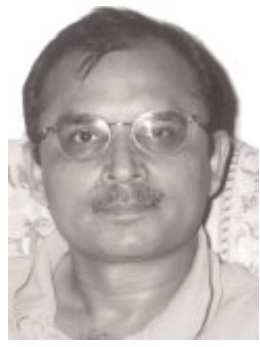

Autar K. Kaw is a professor of mechanical engineering and Jerome Krivanek Distinguished Teacher at the University of South Florida. He is the author of the textbookMechanics of Composite Materials, CRCLLC Press. With major funding from the National Science Foundation, he is developing award winning web-based resources for an undergraduate course in Numerical Methods. He is the recipient of the 2004 CASE Florida Professor of the Year and the 2003 ASEE Archie Higdon Distinguished Mechanics Educator Award. His current scholarly interests include development of instructional technologies, integrating research in classroom, thermal stresses, computational mechanics, and mechanics of nonhomogeneous nanolayers.

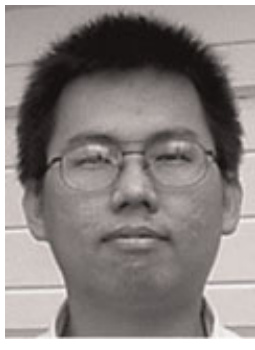

Son H. Ho is a doctoral student in the Department of Mechanical Engineering at the University of South Florida. He received his bachelor's degree with honors in 1995 from the Ho Chi Minh City University of Technology, Viet Nam, and worked there as a research engineer for 6 years. He received his master's degree in 2004 from the University of South Florida. $\mathrm{He}$ is skilled in scientific computer programming with Maple and Matlab. 